\title{
Sensing and Modeling Human Networks using the Sociometer
}

\author{
Tanzeem Choudhury and Alex Pentland \\ Human Design Group \\ 20 Ames Street, E15-384C \\ Cambridge, MA02139 USA \\ $+16172530370$ \\ tanzeem@media.mit.edu
}

\begin{abstract}
Knowledge of how people interact is important in many disciplines, e.g. organizational behavior, social network analysis, information diffusion and knowledge management applications. We are developing methods to automatically and unobtrusively learn the social network structures that arise within human groups based on wearable sensors. At present researchers mainly have to rely on questionnaires, surveys or diaries in order to obtain data on physical interactions between people. In this paper, we show how sensor measurements from the sociometer can be used to build computational models of group interactions. We present results on how we can learn the structure of faceto-face interactions within groups, detect when members are in face-to-face proximity and also when they are having a conversation.
\end{abstract}

\section{Keywords}

Organizational behavior, social network analysis, expertise networks, wearable computing, Bayesian networks.

\section{Introduction}

In almost any social and work situation our decisionmaking is influenced by the actions of others around us. Who are the people we talk to? How often do we talk to them and how long do the conversations last? How actively do we participate in those conversations? Answers to these questions have been used to understand the success and effectiveness of a work group or an organization as a whole. Can we identify the differences between people's interactions? Can we identify the individuals who talk to a large fraction of the group or community members? Such individuals, often referred to the connectors, have an important role in information diffusion [1]. Thus, learning the connection structure and nature of communication among people are important in trying to understand the following phenomena: (i) diffusion of information (ii) group problem solving (iii) consensus building (iv) coalition formation etc. Although people heavily rely on email, telephone and other virtual means of communication, research shows that high complexity information is mostly exchanged through face-to-face interaction [2]. Informal networks of collaboration within organizations coexist with the formal structure of the institution and can enhance the productivity of the formal organization [3]. Furthermore, the physical structure of an institution can either hinder or encourage communication. Usually the probability that two people communicate declines rapidly with the distance between their work locations [2, 4].

We believe the best way to learn informal networks is through observations. We then need to have a mechanism to understand how individuals interact with each other from these observations. Data-driven approach can augment and complement existing manual techniques for data collection and analysis. The goal of our research is twofold - (i) build systems and sensors that can play the role of a mythical "familiar" that sits perched on a user's shoulder, seeing what he sees, with the opportunity to learn what he learns (ii) build an algorithmic pipeline that can take these sensors data and model the dynamics and interconnections between different players in the community. We hope to lay the groundwork for being able to automatically study how different groups within social or business institutions connect. This will help in understanding how information propagates between groups. The knowledge of people's communication networks can also be used in improving context-aware computing environments and coordinating collaboration between group and community members.

\section{Sensing and Modeling Human Communication Networks}

As far as we know, there has been no previous work on modeling face-to-face interactions within a community. This absence is probably due to the difficulty in obtaining reliable measurements from real-world interactions. One has to overcome the uncertainty in sensor measurements, this is in contrast to modeling virtual communities where we can get unambiguous measurements about how people interact - the duration and frequency (available from chat 
and email $\log$ ) and sometime even detailed transcription of interactions $[5,6]$.

We believe sensing and modeling physical interactions among people is an untapped resource. In this paper, we present statistical learning methods that use wearable sensor data to make reliable estimates about a user's interaction state (e.g. who is she talking to, how long did the conversation last, etc.). We use these results to infer the structure/connections that exists in groups of people. This can be much cheaper and more reliable than humandelivered questionnaires. Discovering face-to-face communication networks automatically will also allow researchers to gather interaction data from larger groups of people. This can potentially remove one of the current bottlenecks in the analysis of human networks: the number of people that can be surveyed using manual techniques. Sensor-based approach is free from recall failures and personal interpretation bias of surveys.

\section{Measuring Interactions using the Sociometer}

In this section we describe how we use wearable sensors to measure interactions. The first step towards reliably measuring communication is to have sensors that can capture interaction features. For example, in order to measure face-to-face interactions we need to know who is talking to whom, the frequency and duration of conversations.

We have conducted an experiment at the MIT Media lab where a group of people agreed to wear the sociometer. The sociometer is wearable sensor package that measures people's interactions. It is an adaptation of the hoarder board, a wearable data acquisition board, designed by the electronic publishing and the wearable computing group at the Media lab, for details on the hardware design please refer to $[7,8]$. While designing the sociometer, we put special emphasis on the following issues: comfort of the wearer, aesthetics, and placement of the sensors. We believe these are important points when it comes to greater user acceptance and reliable sensor measurements [9]. The design of the device follows closely the wearability criterion specified in [10], which explores the interaction between the human body and a wearable and provides a guideline on shape and placement of wearables that are unobtrusive and do not interfere with the natural movement of the body.

During the data collection phase, the users had the device on them for six hours a day (11AM -5PM) while they are on the MIT campus. We performed the experiment in two stages - (i) single group stage where 8 subjects from the same research group wore the sociometer for 10 days (60 hours of data per subject) and (ii) multi-group stage where 23 subjects from 4 different research group wore the sociometer for 11 days (over two full work weeks and 66 hours of data per subject). The subjects were a representative sample of the community, including students, faculty and administrative staff

The sociometer has an IR transceiver, a microphone, two accelerometers, on-board storage, and power supply. The wearable stores the data locally on a $256 \mathrm{MB}$ compact flash card and is powered by four AAA batteries. A set of four AAA batteries is enough to power the device for 24 hours. Everything is packaged into a shoulder mount so that it can be worn all day without any discomfort.

The sociometer stores the following information for each individual:

(i) Information about people nearby (sampling rate $17 \mathrm{~Hz}$ - sensor IR)

(ii) Speech information $(8 \mathrm{KHz}-$ microphone)

(iii) Motion information $(50 \mathrm{~Hz}-$ accelerometer $)$

Other sensors (e.g. light sensors, GPS etc.) can also be added in the future using the extension board. For this paper we do not use the data obtained from the accelerometer.

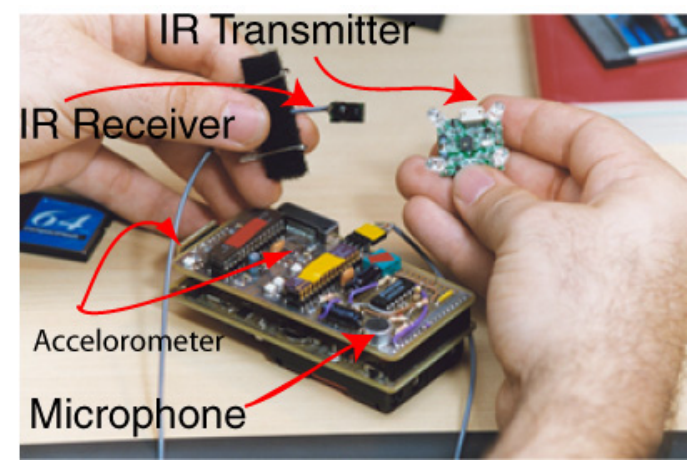

Figure 1 - The wearable sensor board

The success of IR detection depends on the line-of-sight between the transmitter-receiver pair. The sociometer has four low powered IR transmitters. The use of low powered IR transmitters is optimal because (i) we only detect people in close proximity as opposed to far apart in a room (as with high-powered IR) and (ii) we detect people who are facing us and not people all around us (as with RF transmitter). The IR transmitters in the sociometer create a cone shaped region in front of the user where other sociometers can pick up the signal. The range of detection is approximately six feet, which is adequate for picking up face-to-face communication. The design and mounting of the sociometer places the microphone six inches below the wearer's mouth, which enables us to get good audio without a headset. The shoulder mounting also prevents clothing and movement noise that one often gets from clipon microphones. Most of the users were very satisfied with the comfortable and aesthetic design of the device. The majority made no complaints about any inconvenience or discomfort from wearing the device for six hours everyday. 
Despite the comfort and convenience of wearing a sociometer, we are aware that subject's privacy is a concern for any study of human interactions. Most people are wary about how this information will be used. To protect the user's privacy we agree only to extract speech features, e.g. energy, and spectral features from the stored audio and never to process the content of the speech. But, to obtain ground truth we need to label the data somehow, i.e. where do the conversations occur in the data and who are the participants in the conversations. Our proposed solution is to use garbled audio instead of the real audio for labeling. Garbling the audio by swapping 100ms of consecutive audio segments makes the audio content unintelligible but maintains the identity and pitch of the speaker [11]. In future versions of the sociometer we will store encrypted audio instead of the audio, which can also prevent unauthorized access to the data.

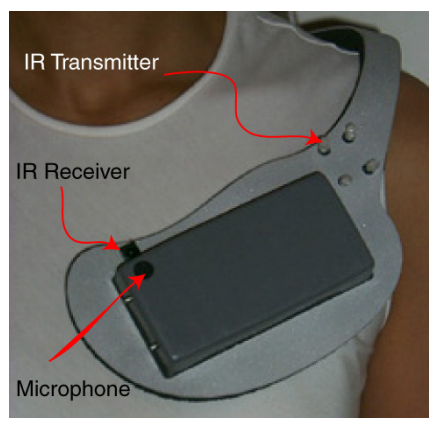

Figure 2 - The shoulder mounted sociometer

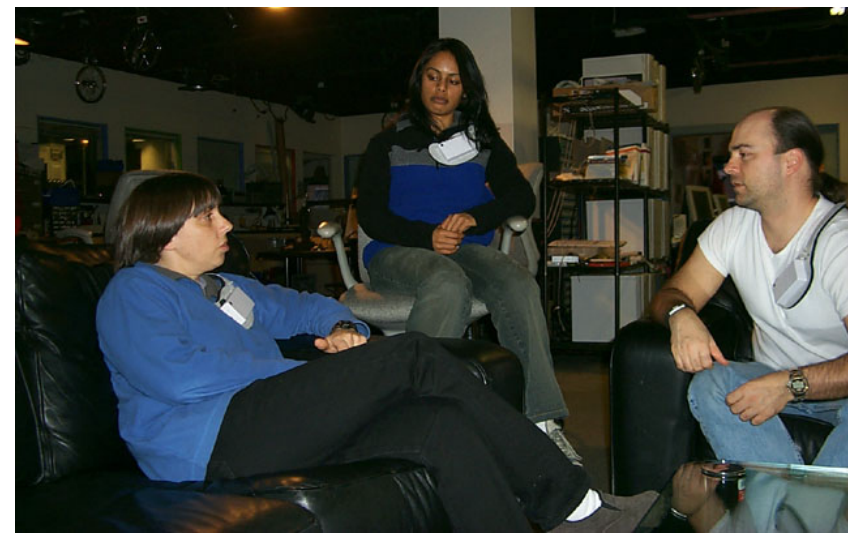

Figure 3 Subjects wearing sociometers during their daily interactions.

\section{Data Analysis Methods}

The first step in the data analysis process is to find out when people are in close proximity. We use the data from the IR receiver to detect proximity of other IR transmitters. The receiver measurements are noisy - the transmitted ID numbers that the IR receivers pick up are not continuous and are often bursty and sporadic. The reason for this bursty signal is that people move around quite a lot when they are talking, so one person's transmitter will not always be within the range of another person's receiver. Consequently, the receiver will not receive the ID number continuously at $17 \mathrm{~Hz}$. Also, each receiver will sometimes receive its self-ID number. We pre-process the IR receiver data by filtering out detection of self ID number as well as propagating one IR receiver information to other nearby receivers (if receiver \#1 detects the presence of tag id \#2, receiver \#2 should also receive tag id \#1). This preprocessing ensures that we maintain consistency between different information channels. However, we still need to identify continuous chunks of time(an episode) when people are in proximity from the bursty receiver measurements. Two episodes are separated by contiguous time chunk in between where no ID is detected. A hidden Markov model (HMM) [12] is trained to learn the pattern of IR signal received over time. Typically an HMM takes noisy observation data (the IR receiver data) and learns the temporal dynamics of the underlying hidden node and its relationship to the observation data. The hidden node in our case has binary state - 1 when the IDs received come from the same episode and 0 when they are from different episodes. We hand-label the hidden states by labeling 6 hours of data. The HMM uses the observation and hidden node labels to learn its parameters. We can now use the trained HMM to assign the most likely hidden states for new observations. From the state labels we can estimate the frequency and the duration that two people are within faceto-face proximity. Figure 4 shows five days of one person's proximity information. Each shade of gray in the sub-image identifies a person to whom the wearer is in close proximity of and the width is the duration contact. Note that we are also able to detect when multiple people are in close proximity at the same time.

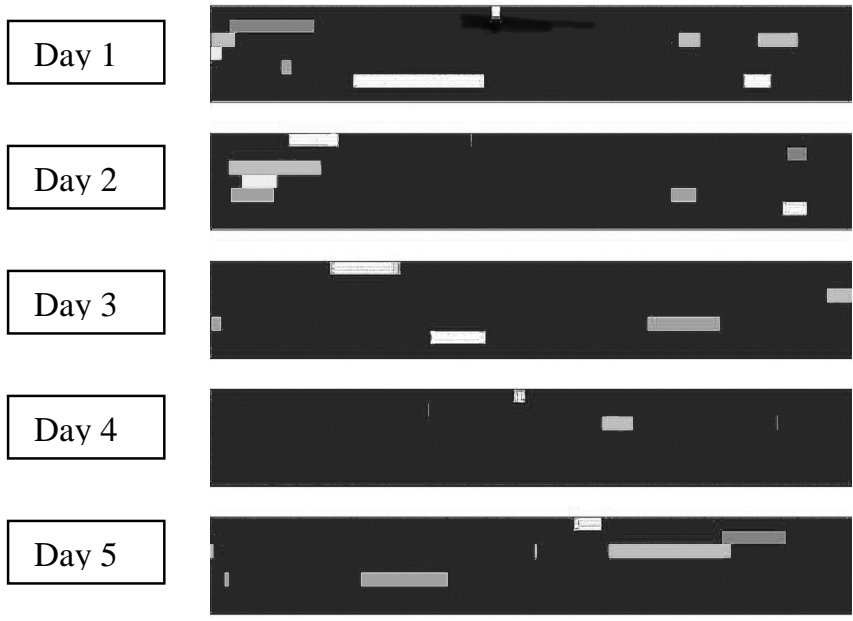

Figure 4 - Proximity information for person 1. Each sub-image shows one day's information. Each row within the sub-image corresponds to a different person. HMM which groups the data into contiguous time chunks. 
The IR tag can provide information about when people are in close face-to-face proximity. But it provides no information about whether two people are actually having a conversation. They may just have been sitting face-to-face during a meeting. In order to identify if two people are actually having a conversation we first need to segment out the speaker from all other ambient noise and other people speaking in the environment. Because of the close placement of the microphone with respect to the speaker's mouth we can use simple energy threshold to segment the speech from most of the other speech and ambient sounds. It is been shown that one can segment speech using voiced regions (speech regions that have pitch) alone [13]. In voiced regions energy is biased towards low-frequency range and hence we use low-energy threshold $(2 \mathrm{KHz}$ cut off) instead of total energy. The output of the lowfrequency energy threshold is passed to another HMM as observation, which segments speech regions from nonspeech regions. The two states of the hidden node correspond to the speech chunks labels $(1=$ a speech region and $0=$ non-speech region). We train our HMM on 10 minutes of speech where the hidden nodes are again hand labeled.

Figure 5 shows the segmentation results for a 35 second audio chunk. In this example two people wearing sociometers are talking to each other and are interrupted by a third person (between $t=20 \mathrm{~s}$ and $\mathrm{t}=30 \mathrm{~s}$ ). The output of low frequency energy threshold for each sociometer is fed into the speech HMM which segments the speech of the wearer. The shaded boxes overlaid on top of the speech signal show the segmentation boundaries for the two speakers. Also note that the third speaker's speech in the 20s-30s region is correctly rejected, as indicated by the grayed region in the figure.

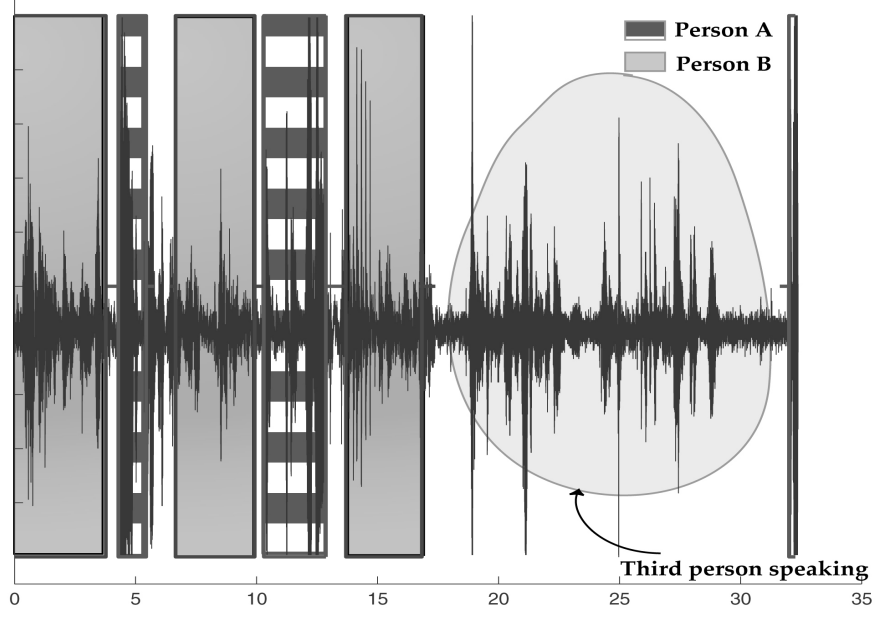

Figure 5 - Speech segmentation for the two subjects wearing the sociometer.

Purely energy-based approach to speaker segmentation is potentially very susceptible to the noise level of the environment and sound from the user's regular activity. In order to overcome this problem we have incorporated robust speech features (non-initial maximum of the autocorrelation, the number of auto-correlation peaks and the normalized spectral entropy) proposed in [13]. An HMM trained to detect voiced/unvoiced regions using these features is very reliable even in noisy environment with less than $2 \%$ error at $10 \mathrm{~dB}$ SSNR. However, the downside of this is any speech and not just the user's speech is detected. So we use a second stage HMM model on the derived features based on energy to segment out only the user's speech and discard all the rest. This method has been very effective in our initial experiments

We now have information about when people are in close proximity and when they are talking. When two people are nearby and talking, it is highly likely that they are talking to each other, but we cannot say this with certainty. Results presented by Basu in [14] demonstrate that we can detect whether two people are in a conversation by relying on the fact that the speech of two people in a conversation is tightly synchronized. We reliably detect when two people are talking to each other by calculating the mutual information of the two voicing streams, which peaks sharply when they are in a conversation as opposed to talking to someone else. The conversation mutual information measure is as follow:

$$
\begin{aligned}
a[k] & =I\left(v_{1}[t], v_{2}[t-k]\right) \\
& =\sum_{i, j} p\left(v_{1}[t]=i, v_{2}[t-k]=j\right) \log \frac{p\left(v_{1}[t]=i, v_{2}[t-k]=j\right)}{p\left(v_{1}[t]=i\right) p\left(v_{2}[t-k]=j\right)}
\end{aligned}
$$

where $\mathrm{v}_{1}$ and $\mathrm{v}_{2}$ are two voicing streams and $\mathrm{i}$ and $\mathrm{j}$ range over 0 and 1 for voiced and unvoiced frames. The performance accuracy in detecting conversations was $63.5 \%$ overall and $87.5 \%$ for conversations greater or equal to one minute. These accuracy numbers were estimated from hand labeled data from four subjects, each of them labeled two days of their data (12 hours each). During the data collection stage we asked the subjects to fill out a daily survey providing a list of their interactions with other members. The survey data had 54\% agreement between subject (where both subjects acknowledged having the conversation) and only $29 \%$ agreement in the number of conversations.

Once we detect the pair-wise conversation chunks we can estimate the duration of conversations. We can further break down the analysis and calculate how long each person talks during a conversation. We can measure the ratio of interaction, i.e. (duration of person A's speech):(duration of person B's speech). We can also calculate what fraction of our total interaction is with people in our community, i.e. inter vs. intra community interactions. This may tell us how embedded a person is within the community vs. how much the person 
communicates with other people. For example, someone who never talks to his work group but has many conversations in general is very different from someone who rarely talks to anyone.

An initial picture of the network structure can be obtained by measuring the duration that people are in close face-to-face proximity using the IR sensor data. Figure 6 shows the link structure of our network based on duration, i.e. the total length of time spent in close proximity. There is an arrow from person $\mathrm{A}$ to person $\mathrm{B}$ if the duration spent in close proximity to B accounts for more than $10 \%$ of A's total time spent with everyone in the network. The thickness of the arrow scales with increasing duration. Similarly, Figure 7 shows the link structure calculated based on frequency, i.e. the number of times two people were in close proximity. We are also in the process of combining both the audio and IR tag information and reestimating the link the structure. In figures 6-8, data from ID4 has not been shown. Although the subject participated in the experiment she was absent for a three days and the clock on her device failed two other days during the data collection. Hence we felt the data from ID4 would not be a representative sample of her interaction with others.

There are a few interesting points to note about differences in the structure based on duration vs. frequency. The two main differences are that in the frequency network there are links between ID \#1 and ID \# 7 and there are extra links connecting ID \#6 to many more nodes than the duration network. The additional link to ID \# 6 was created because person \# 6 sat mostly in the common space through which every one passed through frequently. Consequently, most other receivers often picked up ID \# 6, but the duration of detection was very short. The links between ID 1 an ID 7 are also interesting - although these two people never had long discussions they quite often talked for short periods of time.

Figure 8 shows the fraction of time each individual spends with other members in the group based on duration and frequency. Person 1 talks to all other members regularly and is the most connected person as well (see Figure 6 and Figure 7). Person 2-6 have more skewed distribution in the amount of time they spend with other members, which means they interact mostly with a select sub-group of people. These are only a few examples of looking at different characteristics of the network. Analysis along various dimensions of interaction is going to be one the main advantage of sensor-based modeling of human communication networks.

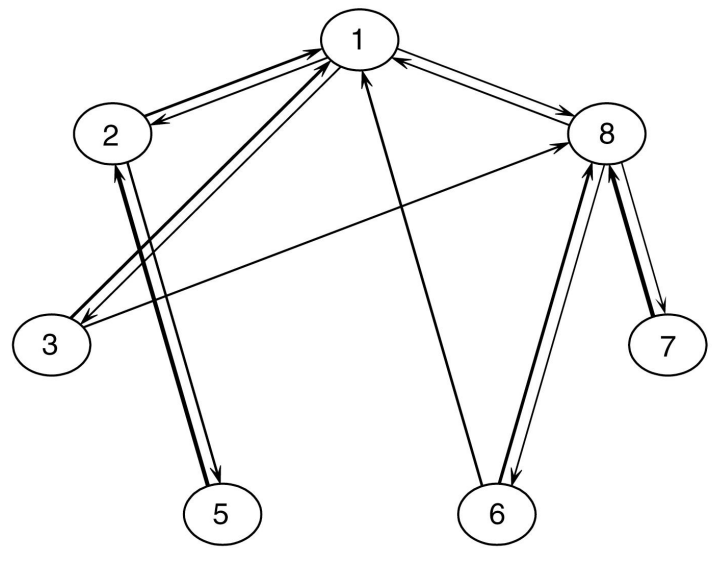

Figure 6 The link structure of the group based on duration.

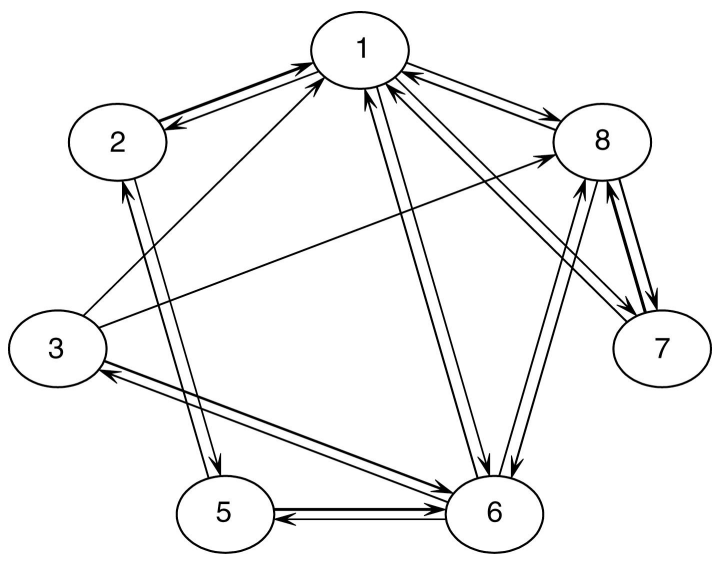

Figure 7 The link structure of the group based on frequency

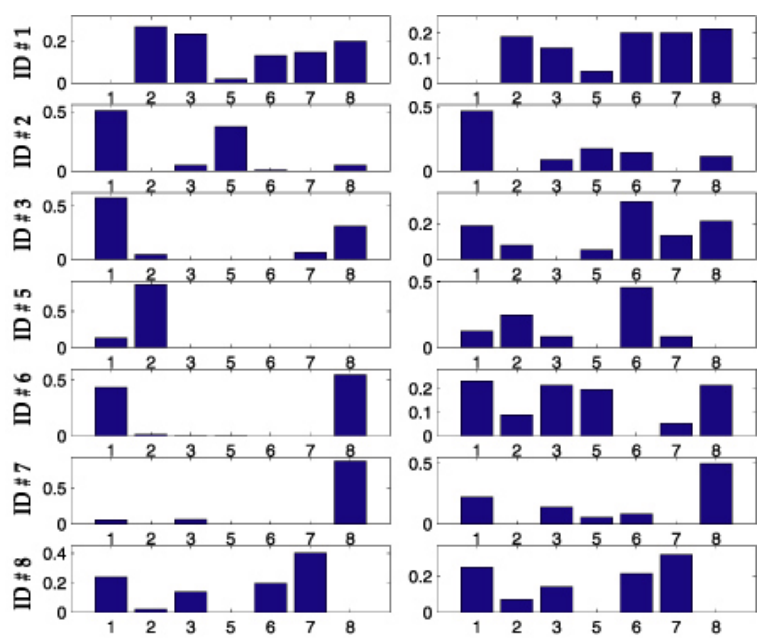

Figure 8 - Interaction distribution based on proximity duration (first column) and proximity frequency (second column). Each row shows results for a different person in the network 
A similar analysis of the larger dataset shows clustering of groups and a reduction of interaction with increasing physical separation. Subject IDs 2-9 belong to group 1, IDs $10,12-15$ belong to group 2, IDs $16-19$ to group 3 and 2124 to group 4, IDs 20 and 25 were physically co-located with groups $1 \& 2$ (no one was assigned ID\# 1 or 11). Note, that there are few individuals that have broad connections across groups (ID 3, 8, and 13) - this type of individuals usually have an important effect on the information flow within the community.

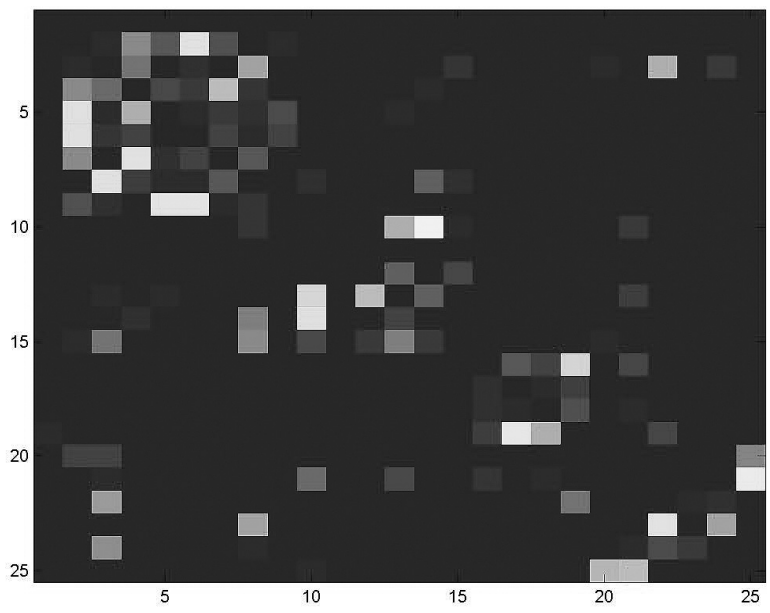

Figure 9 The connectivity matrix of interaction duration. Each row is a different individual and each column depicts the fraction of his/her interaction with others. Image $(i, j)$ depicts person i's interaction with person $j$. Dark region signify absence of interaction.

These connectivity graph or network graph can then be used to estimate centrality measures as traditionally done in social network analysis. Centrality measures seek to quantify an individual's prominence within a network by summarizing the relationships among the different individuals in the network. There are different measures of centrality e.g. degree, betweenness, eigenvector etc.[15, 16]. Here we use the eigenvector centrality where the status of a person is recursively related to the statuses of the people he/she is connected to. If an individual is chosen by a popular person it should add to the person's popularity. If $A$ is the adjacency matrix where $a_{i j}$ means that $I$ contributes to the status of $\mathrm{j}$ and $\mathrm{x}$ is the vector of centrality score - the most general form of eigenvector centrality is:

$$
x_{i}=a_{1 i} x_{1}+a_{2 i} x_{2}+\ldots+a_{n i} x_{n}
$$

In matrix representation:

$$
A^{t} x=x
$$

The eigenvector centrality is the eigenvector of the adjacency matrix corresponding to an eigenvalue of 1 . Normalizing the rows of $A$ to sum to 1 ensures that equation 2 is solvable. The eigenvector centrality measure for the larger group based on the adjacency matrix is shown below (Figure 10), ID 3 and 8 with highest centrality scores also are individuals who had most connection across groups.

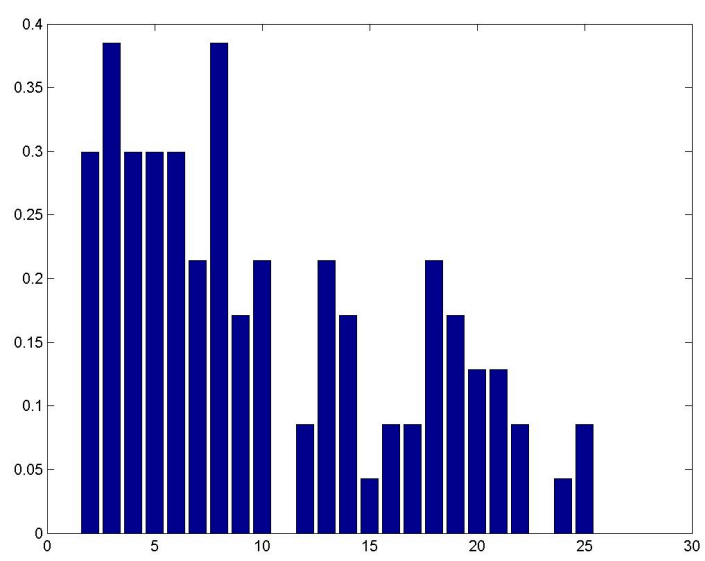

Figure 10 Eigenvector centrality measures of the 23 individual participating in the larger study calculated from proximity data

\section{Conclusion and Future Work}

In this paper, we present a method for analyzing the connectivity of interacting groups using data gathered from wearable sensors. We have presented initial results from our efforts in sensor-based modeling of human communication networks. We show that we can automatically and reliably estimate when people are in close proximity and when they are talking. We demonstrate the advantage of continuous sensing of interactions that allows us to measure the structure of communication networks along various dimensions duration, frequency, ratio of interaction etc. We also present centrality scores for each individuals computed automatically from raw sensor data. Centrality measures are often used in social network analysis as a measure of influence and embeddedness of a person in his/her community. In many studies it has been shown that topology of people's connectivity is the most important feature and the actual interaction content is not as crucial in understanding a person's role within the community[1, 1719]. We are currently obtaining quantitative results for our algorithms by comparing the accuracy of our techniques to hand-labeled ground truth data of the interactions. We are also incorporating our work on modeling the dynamics of the network as a whole that will in the future allow us to quantitatively measure influences people have on each other [20]. 


\section{Acknowledgements}

This work has been partially supported by Center for Bits and Atoms NSF research grant number NSF CCR0122419. The authors would like to especially thank Brian Clarkson for helping with design of the shoulder mount for the sociometer. Thanks to Sumit Basu whose work on Conversational Scene Analysis has guided our work on audio processing. Also thanks to Leonardo Villarreal who spent many hours with me making and testing the 25 sociometers.

\section{REFERENCES}

1. Gladwell, M., The Tipping Point: How little things make can make a big difference. 2000, New York: Little Brown.

2. Allen, T., Architecture and Communication Among Product Development Engineers, 1997, Sloan School of Management, MIT, WP Number 165-97

3. Huberman, B. and Hogg, T., Communities of Practice: Performance and Evolution. Computational and Mathematical Organization Theory, 1995. 1: p. 73-95.

4. Allen, T., Organizational Structure for Product Development, 2000, Sloan School of Management, MIT, WP Number 166-97

5. Gibson, D., Kleinberg, J., and Raghavan, P. Inferring Web Communities from Link Topology. In 9th ACM Conference on Hypertext and Hypermedia. 1998.

6. Lukose, R., Adar, E., Tyler, J., and Sengupta, C. SHOCK: Communicating with Computational Messages and Automatic Private Profiles. In Proceedings of the Twelfth International World Wide Web Conference. 2003.

7. Gerasimov, V., Selker, T., and Bender, W., Sensing and Effecting Environment with Extremity Computing Devices. Motorola Offspring, 2002. 1(1).

8. DeVaul, R. and Weaver, J., MIT Wearable Computing Group. 2002. http://www.media.mit.edu/wearables/.

9. Choudhury, T. and Clarkson, B., Reference Design for A Social Interaction Sensing Platform,, M.L.I.D. Document, Editor. 2002: Cambridge.
10. Gemperle, F., Kasabach, C., Stivoric, J., Bauer, M., and Martin, R., Design for Wearability. 1998, Institute for Complex Engineered Systems, CMU. http://www.ices.cmu.edu/design/wearability/files/Wearabilit y.pdf.

11. Marti, S., Sawhney, N., Jacknis, M., and Schmandt, C., Garble Phone: Auditory Lurking. 2001. http://www.media.mit.edu/speech/projects/garblephone.html

12. Jordan, M. and Bishop, C., An Introduction to Graphical Models. In press: MIT Press.

13. Basu, S. A two layer model for voicing and speech detection. In Conference on Accountics, Speech and Signal Processing (ICASSP). 2003.

14. Basu, S., Conversation Scene Analysis, in Dept. of Electrical Engineering and Computer science. Doctoral. 2002, MIT. p. 1-109.

15. Wasserman, S. and Faust, K., Social Network Analysis Methods and Applications. 1994: Cambridge University Press.

16. Bonacich, P., Power and centrality: a family of measures. American Journal of Sociology, 1987. 92: p. 1170-1182.

17. Tyler, J., D. Wilkinson, and B. Huberman. Email as spectroscopy: Automated discovery of community structure within organizations. in International Conference on Communities and Technologies. 2003. Amsterdam, The Netherlands.

18. Granovetter, M., The strength of weak ties. American Journal of Sociology, 1973. 78(6): p. 1360-1380.

19. Watts, D., Six Degress: The Science of a Connected Age. 2003: W. W. Norton \& Company.

20. Choudhury, T., Clarkson, B., Basu, S., and Pentland, A. Learning Communities: Connectivity and Dynamics of Interacting Agents. In To appear in the proceedings of the International Joint Conference on Neural Networks Special Session on on Autonomous Mental Development. 2003. Portland, Oregon. 